\title{
BMJ Open Electronic cigarette use among adult population: a cross-sectional study in Barcelona, Spain (2013-2014)
}

\author{
Jose M Martínez-Sánchez, ${ }^{1,2,3,4}$ Montse Ballbè, ${ }^{1,2,5,6}$ Marcela Fu, ${ }^{1,2,5}$ \\ Juan Carlos Martín-Sánchez, ${ }^{3}$ Esteve Saltó,,${ }^{7,8}$ Mark Gottlieb, ${ }^{4}$ Richard Daynard, ${ }^{4}$ \\ Gregory N Connolly, ${ }^{9}$ Esteve Fernández ${ }^{1,2,5}$
}

To cite: Martínez-

Sánchez JM, Ballbè M, Fu M, et al. Electronic cigarette use among adult population: a cross-sectional study in Barcelona, Spain (20132014). BMJ Open 2014;4: e005894. doi:10.1136/ bmjopen-2014-005894

- Prepublication history for this paper is available online. To view these files please visit the journal online (http://dx.doi.org/10.1136/ bmjopen-2014-005894).

Received 10 June 2014 Revised 29 July 2014 Accepted 4 August 2014

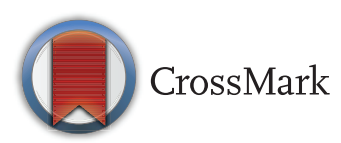

For numbered affiliations see end of article.

Correspondence to Dr Jose M Martínez-Sánchez; jmmartinez@iconcologia.net

\section{ABSTRACT}

Objective: This study seeks to analyse the prevalence and correlates of electronic cigarette (e-cigarette) use, purchase location and satisfaction with its use in a sample of the general population of the city of Barcelona, Spain.

Design, setting and participants: We used participants from a longitudinal study of a representative sample of the adult ( $\geq 16$ years old) population of Barcelona (336 men and 400 women). The field work was conducted between May 2013 and February 2014. We computed the prevalence, adjusted odds ratios (ORs) and their corresponding 95\% confidence interval $(\mathrm{Cl})$.

Results: The prevalence of ever e-cigarette use was $6.5 \%$ ( $95 \% \mathrm{Cl} 4.7 \%$ to $8.3 \%$ ): $1.6 \%$ current use, $2.2 \%$ past use and $2.7 \%$ only e-cigarette experimentation. $75 \%(95 \% \mathrm{Cl} 62.8 \%$ to $87.3 \%)$ of ever e-cigarette users were current cigarette smokers at the moment of the interview. E-cigarette use was more likely among current smokers (OR=54.57; 95\% $\mathrm{Cl} 7.33$ to 406.38) and highly dependent cigarette smokers $(\mathrm{OR}=3.96$; $95 \% \mathrm{Cl} 1.60$ to 9.82 ). $62.5 \%$ of the ever users charged their e-cigarettes with nicotine with $70 \%$ of them obtaining the liquids with nicotine in a specialised shop. $39.6 \%$ of ever e-cigarette users were not satisfied with their use, a similar percentage of not satisfied expressing the smokers (38.9\%) and there were no statistically significant differences in the satisfaction between the users of e-cigarettes with and without nicotine.

Conclusions: E-cigarette use is strongly associated with current smoking (dual use) and most users continue to be addicted to nicotine. Six out of 10 e-cigarette users preferred devices that deliver nicotine. The satisfaction with e-cigarette use is very low.

\section{INTRODUCTION}

The electronic cigarette, also called 'e-cigarette' or electronic nicotine delivery system, is an electronic device commonly shaped like a cigarette. There are also devices resembling cigars or pipes.

\section{Strengths and limitations of this study}

Scientific evidence concerning e-cigarettes is still very limited, particularly in Europe.

- This is the first study to estimate the correlates of use of e-cigarettes in a representative sample of the general adult population in a Mediterranean city.

- The main limitation of our study is attrition of the cohort used, which could lead to a possible no participation bias.

- This is a cross-sectional study and it is only possible to assess associations, but not causal relationships.

- This is the first study that used a face-to-face questionnaire with trained interviewers to assess e-cigarette use in a representative sample of the general population, thus potentially increasing the internal validity of our results.

Regardless of their shape, they are designed to vapourise a mixture of nicotine, propylene glycol and other chemicals that heat the vapour via a battery activated by puffing, but contain no actual tobacco. ${ }^{12}$ The device can also vapourise a mixture that does not contain nicotine. Interest in e-cigarettes has been growing recently among smokers, manufacturers, including leading tobacco companies, and also among tobacco control researchers and the public health community in general, who are concerned with their potential risks and cautiously optimistic about their potential benefits. ${ }^{2}$ Interest in e-cigarettes, as measured by internet searches, exceeded that of snus or nicotine replacement therapies. ${ }^{3}$

Initially, e-cigarettes were primarily obtained through internet sources. Specialty shops and varieties of brands have grown rapidly in recent years in several developed countries. General awareness of e-cigarettes has doubled in just 1 year in the USA. ${ }^{4}$ However, scientific evidence concerning 
e-cigarettes is still very limited, including valid estimates of the prevalence of e-cigarette use among the general population, particularly in Europe. Moreover, to our knowledge, there is a lack of information on specific characteristics of use, such as the location of purchase, use of liquids containing nicotine and the satisfaction with this product among users. These issues are especially relevant to characterise the use of this new product in order to implement future regulations.

The objective of this study is to estimate the prevalence and analyse the correlates of current and ever use of e-cigarettes, including purchase location and satisfaction with its use, in a sample of the general population of the city of Barcelona (Spain) in 2013 and 2014.

\section{METHODS}

The Determinants of Cotinine phase 3 project (dCOT3, website: http://bioinfo.iconcologia.net/es/content/ estudio-dcot3) is a longitudinal study of a representative sample of the adult ( $\geq 16$ years old) non-institutionalised population of the city of Barcelona (Spain; n=1245, 694 women and $551 \mathrm{men})$. The theoretical baseline sample size was 1291 individuals, assuming an expected smoking prevalence of $30 \%$ (with an $\alpha$ error of $5 \%$ and a precision of $2.5 \%$ ), which was the estimated percentage of smokers in Spain when the baseline survey was conducted. The baseline survey was conducted in 20042005 and its detailed design has been provided elsewhere. ${ }^{5}{ }^{6}$ We followed up all the adult participants who responded to a face-to-face questionnaire in 2004-2005 and agreed to participate in future studies. At the beginning of 2013, we did a linkage with the Insured Central Registry of Catalonia in order to update the vital status and contact information (addresses and telephone numbers) of all participants. We restricted the follow-up to the participants who continued to live in the city of Barcelona and their province.

We traced 1010 people out of the 1245 participants in the baseline study using the Insured Central Registry (101 have died, 49 migrated out of the province of Barcelona and 85 did not give consent to be followed or were $<18$ years old in 2004-2005). In February 2013, we sent them a letter stating the primary findings of the 2004-2005 study and informed them that an interviewer would go to their home to administer another face-to-face questionnaire. The follow-up survey was conducted between May 2013 and February of 2014. In total, $72.9 \%$ agreed to participate and responded to the questionnaire, $18.5 \%$ refused to participate in the follow-up, $7.2 \%$ had moved elsewhere and $1.3 \%$ had died. The final sample analysed was 736 individuals (336 men and 400 women). Although there were no statistically significant differences between the followed up sample and the participants lost according to age, sex, level of education and smoking status, the final sample skewed as slighter older (data not shown).
Data on current use, ever use and experimentation with e-cigarettes were obtained using the question (as translated from Spanish): "Have you ever used e-cigarettes?" The answers to this question were: "yes, currently"; "yes, in the past"; "I have only experimented with e-cigarettes"; and "I have never used e-cigarettes." We also included two questions about the use of e-cigarettes with or without nicotine using the question: "Do/did you use the electronic cigarettes with nicotine?" (yes/no) and the places where the nicotine was obtained (internet, specialised shop or other countries). Finally, we asked ever e-cigarette users about their satisfaction with e-cigarettes using the question: "How satisfied are you with the use of the electronic cigarette?" The possible answers for this question were: "totally satisfied"; "quite satisfied"; "somewhat satisfied" and "not satisfied" (recoded as "totally and quite satisfied," "somewhat" and "not satisfied"). We calculated the prevalence and the adjusted odds ratios (ORs) with 95\% confidence intervals (CIs) by sex, age and educational level. All analyses were stratified by sex; groups of age ( $\leq 44,45-64$ and $\geq 65$ years old); educational level-categorised as low (no qualification up to middle school diploma), intermediate (high school) and high (university degree); cigarette smoking status (current smokers as participants who smoked cigarettes either daily (at least one cigarette/day) or occasionally (less than one cigarette/day) at the moment of the survey, former smokers as participant who did not smoke cigarettes at the moment of the survey but had smoked cigarettes in the past and never-smokers as participants who have never smoked cigarettes); and level of nicotine dependence measured with the Fagerström test for cigarette dependence $(\mathrm{FTCD})^{7}$ for current cigarette smokers, and categorised into low-medium dependence for scores between 0 and 5 and high dependence for scores between 6 and 10 .

\section{RESULTS}

The prevalence of smokers of manufactured cigarettes was $23.3 \%$ (95\% CI $20.2 \%$ to $26.3 \%$ ) and the prevalence of ever e-cigarette use was $6.5 \%$ (95\% CI $4.7 \%$ to $8.3 \%$ ). Smokers of manufactured cigarettes had a mean age of 49.4 years, $53.8 \%$ were men and $47.9 \%$ had intermediate educational level. The e-cigarette users had a mean age of 45.1 years, $56.2 \%$ were men and $58.3 \%$ had intermediate educational level. There were no statistically significant differences according to demographic characteristics (sex, age, and level of education) between smokers of manufactured cigarettes and e-cigarette users. In total, $75 \%$ of e-cigarette users were smokers, $22.9 \%$ were former smokers and $2.1 \%$ were never-smokers at the time of the interview. The prevalence of ever e-cigarette use was higher among men $(8 \%)$, younger people ( $\leq 44$ years old, $13.1 \%)$ and people with intermediate educational level (9.8\%, $\mathrm{OR}=1.42,95 \%$ CI 0.50 to 4.04 ). There was a statistically 
significant association between ever e-cigarette use and current smoking (OR=54.57, 95\% CI 7.33 to 406.38). Also, the highest prevalence $(46.4 \%)$ of ever e-cigarette use was among current smokers with a high cigarette dependence score (table 1 ).

In total, $62.5 \%$ of ever e-cigarette users $(95 \%$ CI $48.8 \%$ to $76.2 \%$ ) used them with nicotine and $70 \%$ (95\% CI $53.6 \%$ to $86.4 \%)$ indicated they obtained the liquid with nicotine in a specialty shop, while $3.3 \%$ (95\% CI $0.6 \%$ to $16.7 \%)$ indicated that they obtained it on the internet. There were no statistically significant differences according to sex, age, educational level and smoking status regarding the use of e-cigarettes with nicotine or not (table 1), and the places where they obtained the liquid with nicotine (data not shown). Among ever e-cigarette users, $18.8 \%(95 \%$ CI $7.7 \%$ to $29.9 \%$ ) were totally or quite satisfied with their use and $39.6 \%$ (95\% CI $25.8 \%$ to $53.4 \%$ ) were not satisfied. The percentage of not satisfied users was $38.9 \%$ among current smokers, $30.8 \%$ among smokers with high cigarette dependence score (table 1). There were no statistically significant differences in the satisfaction with e-cigarettes according to use of liquids with and without nicotine (not satisfied: $40 \%$ vs $38.9 \%$; OR $=0.53,95 \%$ CI 0.11 to 2.49$)$.

Table 2 shows the prevalence rates of current use, past use and only experimentation with e-cigarettes. In total,
$1.6 \%$ were current users, $2.2 \%$ past users and $2.7 \%$ had only experimented with e-cigarettes. There were no statistically significant differences among current e-cigarette users according to sex, age and educational level. Finally, the prevalence of current use was higher among current smokers (dual users) and among current smokers with high cigarette dependence score $(5.3 \%$ and $14.3 \%$, respectively).

\section{DISCUSSION}

This is the first study to estimate the use of e-cigarettes in a representative sample of the general adult population in a Mediterranean city. The prevalence of ever e-cigarette use was $6.5 \%$ (1.6\% current use, $2.2 \%$ past use and $2.7 \%$ only e-cigarette experimentation) and the predominant ever and current e-cigarette use was among current smokers (75\% of ever e-cigarette users were current smokers). Similar results were found for the general population in Europe according to the Eurobarometer survey conducted in $2012^{8}$ and also in the $\mathrm{USA}^{9}$ according to a study conducted in 2010-2011. Surprisingly, our prevalence of ever use is lower to what we would expect, considering the increase of marketing and popularity of e-cigarettes in recent years. This low prevalence could be due to a potential delay in the general marketing of e-cigarettes in Spain as compared

Table 1 Prevalence of ever e-cigarette use, percentage of users of e-cigarettes with cotinine, and percentage of satisfaction with e-cigarettes according to sex, age, educational level, smoking status and FTCD in Barcelona, Spain (2013-14).

\begin{tabular}{|c|c|c|c|c|c|c|c|c|}
\hline & \multirow[b]{2}{*}{$\mathbf{n}$} & \multicolumn{2}{|c|}{ Ever e-cigarette users } & \multirow[b]{2}{*}{$\mathbf{n}$} & \multicolumn{2}{|c|}{$\begin{array}{l}\text { Ever use of e-cigarettes } \\
\text { with nicotine }\end{array}$} & \multicolumn{2}{|c|}{$\begin{array}{l}\text { Satisfaction with the } \\
\text { usage of e-cigarettes } \\
\text { (not satisfied) }\end{array}$} \\
\hline & & $\%$ & $\mathrm{OR}^{\star}(95 \% \mathrm{Cl})$ & & \%† & $\mathrm{OR}^{\star}(95 \% \mathrm{Cl})$ & $\% †$ & $\mathrm{OR}^{\star}(95 \% \mathrm{Cl})$ \\
\hline Overall & 736 & 6.5 & & 48 & 62.5 & & 39.6 & \\
\hline \multicolumn{9}{|l|}{ Sex } \\
\hline Men & 336 & 8.0 & 1 & 27 & 51.9 & 1 & 44.4 & 1 \\
\hline$\leq 44$ & 198 & 13.1 & 1 & 26 & 73.1 & 1 & 30.8 & 1 \\
\hline $45-64$ & 267 & 6.7 & $0.39(0.20$ to 0.75$)$ & 18 & 44.4 & 0.32 (0.08 to 1.28$)$ & 55.6 & $3.21(0.78$ to 13.13$)$ \\
\hline$\geq 65$ & 271 & 1.5 & $0.08(0.02$ to 0.24$)$ & 4 & 75.0 & $1.49(0.13$ to 17.48$)$ & 25.0 & $0.73(0.05$ to 10.84$)$ \\
\hline \multicolumn{9}{|l|}{ Educational level } \\
\hline Low & 161 & 3.1 & 1 & 5 & 60.0 & 1 & 0.0 & - \\
\hline Former smoker & 267 & 4.1 & $13.19(1.68$ to 103.82$)$ & 11 & 54.5 & 1 & 45.5 & 1 \\
\hline Current smoker & 171 & 21.1 & 54.57 (7.33 to 406.38$)$ & 36 & 66.7 & $1.22(0.21$ to 6.92$)$ & 38.9 & $1.30(0.28$ to 5.96$)$ \\
\hline \multicolumn{9}{|l|}{ FTCD } \\
\hline Low-Medium (0-5) & 143 & 16.1 & 1 & 23 & 60.9 & 1 & 43.5 & 1 \\
\hline High $(6-10)$ & 28 & 46.4 & 3.96 (1.60 to 9.82$)$ & 13 & 76.9 & $5.86(0.73$ to 46.77$)$ & 30.8 & 0.14 (0.01 to 1.42$)$ \\
\hline $\begin{array}{l}\text { *Adjusted ORs for sex, a } \\
\text { †Prevalence among ever } \\
\text { FTCD, Fagerström test fo } \\
\text { OR, odds ratio; Cl, confid }\end{array}$ & $\begin{array}{l}\text { e and } \\
\text { e-cigar } \\
\text { cigare }\end{array}$ & $\begin{array}{l}\text { ducatio } \\
\text { tte use } \\
\text { te depe } \\
\text { ervals. }\end{array}$ & $\begin{array}{l}\text { hal level. } \\
\text { s. } \\
\text { ndence. }\end{array}$ & & & & & \\
\hline
\end{tabular}


Table 2 Prevalence of current use, past use and experimentation with e-cigarettes according to sex, age, educational level, smoking status and FTCD in Barcelona, Spain (2013-14).

\begin{tabular}{|c|c|c|c|c|}
\hline & $\mathbf{n}$ & $\begin{array}{l}\text { Current } \\
\text { e-cigarette use } \\
\%(95 \% \mathrm{Cl})\end{array}$ & $\begin{array}{l}\text { Past } \\
\text { e-cigarette use } \\
\%(95 \% \mathrm{Cl})\end{array}$ & $\begin{array}{l}\text { Experimentation with } \\
\text { e-cigarettes } \\
\%(95 \% \mathrm{Cl})\end{array}$ \\
\hline Overall & 736 & $1.6(0.7$ to 2.5$)$ & 2.2 (1.1 to 3.3$)$ & 2.7 (1.5 to 3.9$)$ \\
\hline \multicolumn{5}{|l|}{ Sex } \\
\hline Men & 336 & 1.8 (0.4 to 3.2$)$ & 3.3 (1.4 to 5.2$)$ & 3.0 (1.2 to 4.8$)$ \\
\hline Women & 400 & 1.5 (0.3 to 2.7$)$ & $1.3(0.2$ to 2.4$)$ & 2.5 (1.0 to 4.0$)$ \\
\hline \multicolumn{5}{|l|}{ Age (years) } \\
\hline$\leq 44$ & 198 & $2.0(0.0$ to 4.0$)$ & 5.1 (2.0 to 8.2$)$ & 6.1 (2.8 to 9.4$)$ \\
\hline $45-64$ & 267 & $1.9(0.3$ to 3.5$)$ & $1.9(0.3$ to 3.5$)$ & $3.0(1.0$ to 5.0$)$ \\
\hline$\geq 65$ & 271 & 1.1 (0.4 to 3.2) & $0.4(0.1$ to 2.1$)$ & $0.0(0.0$ to 1.4$)$ \\
\hline \multicolumn{5}{|l|}{ Educational level } \\
\hline Low & 161 & $1.2(0.3$ to 4.4$)$ & 0.0 (0.0 to 2.3$)$ & 1.9 (0.6 to 5.3$)$ \\
\hline Intermediate & 287 & 2.4 (0.6 to 4.2$)$ & 4.5 (2.1 to 6.9$)$ & $2.8(0.9$ to 4.7$)$ \\
\hline High & 288 & $1.0(0.4$ to 3.0$)$ & $1.0(0.4$ to 3.0$)$ & 3.1 (1.1 to 5.1$)$ \\
\hline \multicolumn{5}{|l|}{ Smoking status } \\
\hline Never-smoker & 298 & 0.3 (1.6 to 5.6$)$ & 0.0 (0.0 to 1.3$)$ & 0.0 (0.0 to 1.3$)$ \\
\hline Former smoker & 267 & 0.7 (0.2 to 2.7$)$ & 1.1 (0.4 to 3.3$)$ & 2.2 (0.4 to 4.0$)$ \\
\hline Current smoker & 171 & 5.3 (1.9 to 8.7$)$ & 7.6 (3.6 to 11.6$)$ & 8.2 (4.1 to 12.3$)$ \\
\hline \multicolumn{5}{|l|}{ FTCD } \\
\hline Low-Medium (0-5) & 143 & 3.5 (0.5 to 6.5$)$ & 5.6 (1.8 to 9.4$)$ & 7 (2.8 to 11.2$)$ \\
\hline High (6-10) & 28 & 14.3 (1.3 to 27.3$)$ & 17.9 (3.7 to 32.1$)$ & 14.3 (1.3 to 27.3$)$ \\
\hline
\end{tabular}

with other countries, as well as the quick reaction of the tobacco control community and public health authorities to apply the precautionary principle in Spain. ${ }^{10} \mathrm{We}$ also found that $62.5 \%$ of the ever e-cigarette users preferred liquids with nicotine and specialty shops were the places where they most frequently bought these liquids $(70 \%)$.

One study conducted among young Swiss men showed lower prevalence of ever e-cigarette use in the past 12 months than in our study $(4.9 \%))^{11}$ A study conducted among teenagers from Poland (between 15 and 24 years old) showed that $6.9 \%$ of them reported experimenting with e-cigarettes in the previous 30 days ${ }^{12}$ while we found $13.1 \%$ of ever e-cigarette use among young people ( $\leq 44$ years old). Another study conducted in the UK, using telephone interviews among current and former smokers, ${ }^{13}$ showed that $4 \%$ were current e-cigarette users and, among those who were aware of e-cigarettes, $17.7 \%$ had tried e-cigarettes, which is slightly lower than in our study $(5.3 \%$ and $21.1 \%$, respectively). However, the differences in the questions used to measure the prevalence of e-cigarette use make the comparison among studies difficult.

Currently, there is an intense debate in the tobacco control community about the usefulness of e-cigarettes as a new strategy to quit or reduce tobacco consumption and its potential harmful health effects. ${ }^{14-22}$ The only clinical trial published to date ${ }^{23}$ showed that $7.3 \%$ of those who used e-cigarettes with nicotine to quit smoking were still abstinent at 6 months, compared with $5.8 \%$ who used nicotine patches and to $4.1 \%$ who used e-cigarettes without nicotine, although no statistically significant differences were found. Two longitudinal studies ${ }^{24} 25$ also found that e-cigarettes may contribute to preventing relapse in former smokers and to promote smoking cessation in current smokers. Other studies ${ }^{49} 26$ suggest that there is a high percentage of e-cigarette users concurrently using conventional tobacco. However, the evidence is still scarce according to recent reviews of the scientific literature. ${ }^{27} 28$ According to our data, we likewise found a high percentage $(75 \%)$ of current e-cigarette users exhibiting dual use patterns with conventional tobacco. Moreover, we surprisingly found a very low percentage of ever e-cigarette users quite or totally satisfied with their use $(18.8 \%)$, particularly among current smokers and smokers with a high score in the FTCD (13.9\% and $7.7 \%$, respectively). Our hypothesis is that these highly nicotinedependent smokers tried e-cigarettes for smoking cessation or to reduce cigarette use, but they continued smoking or relapsed in a short time. In addition, we found no differences in the satisfaction according to the use of the e-cigarettes with or without nicotine. More longitudinal and qualitative studies are needed to confirm this hypothesis.

Some studies suggest that e-cigarettes could be another way to create new nicotine addicts, ${ }^{29} 30$ particularly among young people, who may graduate to conventional tobacco products over time. Moreover, the current advertisements and messages about e-cigarettes in the media and the social networks, such as twitter, could increase the experimentation, particularly among the young and middle aged population. ${ }^{31-33}$ The results of 
our study show that $62.5 \%$ of ever e-cigarette users preferred e-cigarettes with nicotine, and a considerable percentage of them were young people. However, the percentage of never-smokers who had ever used the e-cigarettes is very low $(0.3 \%)$ and its use was without nicotine. However, this result should be taken with caution because of the small sample size in this category.

The main limitation of our study is the potential of participation bias due to the attrition of the cohort of participants. Although there are no statistically significant differences between the people who were followed up and those lost from the original study according to sex, age and educational level, our final sample overestimated the older people compared with the distribution of population in Barcelona. For this reason, the prevalence of e-cigarette use might be underestimated in our study because young people, particularly younger smokers, are those who most used e-cigarettes. Moreover, we conducted the study only in the city of Barcelona and the validity to infer the results to the rest of Spain could be limited. Nevertheless, the baseline sample size was representative of the city of Barcelona ${ }^{5}{ }^{6}$ and the final sample size for this analysis was sufficient to estimate the prevalence of e-cigarette users, due to the relatively lower prevalence of ever e-cigarette use in the general population. ${ }^{8}$ According to an expected prevalence of ever e-cigarette use of $10 \%$, the sample size would be 554 individuals, with an $\alpha$ error of $5 \%$ and a precision of $2.5 \%$. Another potential limitation is the use of a questionnaire to collect self-reported information on e-cigarette use that could be an inherent source of bias. However, this is the first study, to our knowledge, that used a face-to-face questionnaire with trained interviewers to assess e-cigarette use in a representative sample of the general population, thus potentially increasing the internal validity of our results as compared with internet and other self-administered surveys. ${ }^{9} 26$ Additionally, our results could slightly underestimate the real prevalence of use, because we only included the term 'e-cigarette' in the questionnaire, and there are other terms associated to new products in the market. However, this effect may be limited, because the term 'e-cigarettes' is the most popular in Spain, and products such as 'hookah pens' or 'vape pens' are scantily marketed. Finally, this is a crosssectional study and it is only possible to assess associations and not causal relationships.

In conclusion, $6.5 \%$ of the adult general populations in Barcelona (Spain) are ever e-cigarette users, and 6 out of 10 of them used devices that deliver nicotine. According to evidence from other countries, this figure could double in coming years among the general population $^{9}$ as well as in the adolescent and student populations. $^{29} 34$ Furthermore, our results show that current and ever e-cigarette use was predominant among current smokers, indicating a dual use pattern, and that there were very low levels of satisfaction with e-cigarettes. More investigation is needed on dual use (e-cigarettes and conventional tobacco) and on users' satisfaction with e-cigarette devices, as well as on the effectiveness of e-cigarettes for smoking cessation and their benefit-risk balance.

Author affiliations

${ }^{1}$ Tobacco Control Unit, Cancer Prevention and Control Program, Institut Català d'Oncologia, L'Hospitalet de Llobregat, Barcelona, Spain

${ }^{2}$ Cancer Prevention and Control Group, Institut d'Investigació Biomèdica de Bellvitge - IDIBELL, L'Hospitalet de Llobregat, Barcelona, Spain

${ }^{3}$ Biostatistics Unit, Department of Basic Sciences, Universitat Internacional de Catalunya, Sant Cugat del Vallès, Barcelona, Spain

${ }^{4}$ Public Health Advocacy Institute, Northeastern University School of Law, Boston, Massachusetts, USA

${ }^{5}$ Department of Clinical Sciences, Universitat de Barcelona, Barcelona, Spain ${ }^{6}$ Addictions Unit, Institute of Neurosciences, Hospital Clínic de BarcelonaIDIBAPS, Barcelona, Spain

${ }^{7}$ Health Plan Directorate, Ministry of Health, Generalitat de Catalunya, Barcelona, Spain

${ }^{8}$ Department of Public Health, Universitat de Barcelona, Barcelona, Spain ${ }^{9}$ Department of Social and Behavioral Sciences, Center for Global Tobacco Control, Harvard School of Public Health, Boston, Massachusetts, USA

Acknowledgements The authors wish to thank Nuria Quirós for her contribution to the follow-up of participants by means of the Insurance Central Registry of Catalonia and Montse Ferré and Lucía Baranda with the coordination of the filed work operations.

Contributors JMMS and EF conceived the study. MB, MF, EF, ES and JMMS contributed to the design and coordination of the study. JCMS analysed the data. JMMS drafted the first manuscript. All authors contributed substantially to the interpretation of the data and the successive versions of the manuscript. All authors contributed to the manuscript and approved its final version. JMMS is the principal investigator of the project.

Funding This project was funded by the Instituto de Salud Carlos III, Government of Spain (RTICC, RD12/0036/0053 and PI12/01114) and the Ministry of Universities and Research, Government of Catalonia (grant 2009SGR192).

\section{Competing interests None.}

Ethics approval The Research and Ethics Committee of the Bellvitge University Hospital provided ethical approval for the study protocol.

Provenance and peer review Not commissioned; externally peer reviewed.

Data sharing statement No additional data are available.

Open Access This is an Open Access article distributed in accordance with the Creative Commons Attribution Non Commercial (CC BY-NC 4.0) license, which permits others to distribute, remix, adapt, build upon this work noncommercially, and license their derivative works on different terms, provided the original work is properly cited and the use is non-commercial. See: http:// creativecommons.org/licenses/by-nc/4.0/

\section{REFERENCES}

1. Etter JF, Bullen C, Flouris AD, et al. Electronic nicotine delivery systems: a research agenda. Tob Control 2011;20:243-8.

2. Cobb NK, Byron MJ, Abrams DB, et al. Novel nicotine delivery systems and public health: the rise of the "e-cigarette". Am J Public Health 2010;100:2340-2.

3. Ayers JW, RibisI KM, Brownstein JS. Tracking the rise in popularity of electronic nicotine delivery systems (electronic cigarettes) using search query surveillance. Am J Prev Med 2011;40:448-53.

4. Regan AK, Promoff G, Dube SR, et al. Electronic nicotine delivery systems: adult use and awareness of the 'e-cigarette' in the USA. Tob Control 2013;22:19-23.

5. Fu M, Fernandez E, Martinez-Sanchez JM, et al. Salivary cotinine concentrations in daily smokers in Barcelona, Spain: a cross-sectional study. BMC Public Health 2009;9:320.

6. Martinez-Sanchez JM, Fernandez E, Fu M, et al. Assessment of exposure to secondhand smoke by questionnaire and salivary 
cotinine in the general population of Barcelona, Spain (2004-2005). Prev Med 2009;48:218-23.

7. Fagerstrom K. Determinants of tobacco use and renaming the FTND to the Fagerstrom Test for Cigarette Dependence. Nicotine Tob Res 2012;14:75-8.

8. European Commision. Attitudes of Europeans towards tobacco (Special Eurobarometer 385). http://ec.europa.eu/health/tobacco/ eurobarometers/index_en.htm (accessed 20 May 2014)

9. King BA, Alam S, Promoff G, et al. Awareness and ever-use of electronic cigarettes among U.S. adults, 2010-2011. Nicotine Tob Res 2013;15:1623-7.

10. Xarxa Catalana d'Hospitals sense fum. Statement of the Catalan Network of Smoke-free Hospitals and the Network of Primary Care on the use of electronic cigarettes in health centers. Barcelona, 30 Jul 2013. http://tobaccorelated.files.wordpress.com/2013/08/ statement_ecigs.pdf (accessed 28 Jul 2014)

11. Douptcheva N, Gmel G, Studer J, et al. Use of electronic cigarettes among young Swiss men. $J$ Epidemiol Community Health 2013;67:1075-6.

12. Goniewicz ML, Zielinska-Danch W. Electronic cigarette use among teenagers and young adults in Poland. Pediatrics 2012;130:e879-85.

13. Adkison SE, O'Connor RJ, Bansal-Travers M, et al. Electronic nicotine delivery systems: international tobacco control four-country survey. Am J Prev Med 2013;44:207-15.

14. Cobb NK, Abrams DB. E-cigarette or drug-delivery device? Regulating novel nicotine products. N Engl J Med 2011;365:193-5.

15. Fairchild AL, Bayer R, Colgrove J. The renormalization of smoking? E-cigarettes and the tobacco "endgame". N Engl J Med 2014;370:293-5.

16. Farsalinos $\mathrm{K}$, Voudris V. It is preferable for surgical patients to use e-cigarettes rather than smoke cigarettes. BMJ 2014;348:g1961.

17. Kmietowicz Z. E-cigarettes are "gateway devices" for smoking among young people, say researchers. BMJ 2014;348:g2034.

18. Auf R. Electronic cigarettes and smoking cessation: a quandary? Lancet 2014;383:408.

19. Bialous SA, Sarma L. Electronic cigarettes and smoking cessation: a quandary? Lancet 2014;383:407-8.

20. Bullen C, Howe C, Laugesen M, et al. Electronic cigarettes and smoking cessation: a quandary?-Authors' reply. Lancet 2014;383:408-9.
21. Doyle C, Patterson S, Scott J. Electronic cigarettes and smoking cessation: a quandary? Lancet 2014;383:408.

22. Hajek P. Electronic cigarettes for smoking cessation. Lancet 2013;382:1614-16.

23. Bullen C, Howe C, Laugesen M, et al. Electronic cigarettes for smoking cessation: a randomised controlled trial. Lancet 2013;382:1629-37.

24. Etter JF, Bullen C. A longitudinal study of electronic cigarette users. Addict Behav 2014;39:491-4.

25. Polosa R, Caponnetto P, Morjaria JB, et al. Effect of an electronic nicotine delivery device (e-Cigarette) on smoking reduction and cessation: a prospective 6-month pilot study. BMC Public Health 2011;11:786.

26. Pearson JL, Richardson A, Niaura RS, et al. e-Cigarette awareness, use, and harm perceptions in US adults. Am J Public Health 2012;102:1758-66.

27. Grana RA, Ling PM, Benowitz N, et al. Electronic cigarettes. Circulation 2014;129:e490-2.

28. Pepper JK, Brewer NT. Electronic nicotine delivery system (electronic cigarette) awareness, use, reactions and beliefs: a systematic review. Tob Control 2014;23:375-84.

29. Dutra LM, Glantz SA. Electronic cigarettes and conventional cigarette use among US adolescents: a cross-sectional Study. JAMA Pediatr 2014 (in press) doi:10.1001/jamapediatrics.2013.5488

30. Lee S, Grana RA, Glantz SA. Electronic cigarette use among Korean adolescents: a cross-sectional study of market penetration, dual use, and relationship to quit attempts and former smoking $J$ Adolesc Health 2014;54:684-90.

31. Pepper JK, Emery SL, Ribisl KM, et al. Effects of advertisements on smokers' interest in trying e-cigarettes: the roles of product comparison and visual cues. Tob Control 2014;23(Suppl 3):iii31-6.

32. Huang J, Kornfield R, Szczypka G, et al. A cross-sectional examination of marketing of electronic cigarettes on Twitter. Tob Control 2014;23(Suppl 3):iii26-30.

33. de Andrade M, Hastings G, Angus K. Promotion of electronic cigarettes: tobacco marketing reinvented? BMJ 2013;347:f7473.

34. Corey C, Wang B, Johnson SE, et al. Centers for Disease Control and Prevention. Notes from the field: electronic cigarette use among middle and high school students-United States, 2011-2012. MMWR Morb Mortal Wkly Rep 2013;62:729-30. 